\title{
Cervical Transverse Mega-apophysis: A Rare Cause of Plexopathy
}

\author{
Pierre Ferrer ${ }^{1}$, Ana S Álvarez ${ }^{2}$, Juan R Penanes ${ }^{3}$
}

\begin{abstract}
Aim: This article aims to describe the case of a 43-year-old male with a neurogenic thoracic outlet syndrome caused by a C7 transverse mega-apophysis.

Background: Cervical transverse mega-apophysis, transverse apophysomegaly, or elongation of the transverse vertebral process represents a variation of normal skeletal anatomy. This variation has been little studied and its prevalence in the population is unknown because it often exists without symptoms. It is estimated that less than $10 \%$ of cases are symptomatic.

Case description: We present a rare case of a man with a neurogenic thoracic outlet syndrome (in this case, a left plexopathy) caused by a cervical transverse mega-apophysis. After surgical intervention, the patient improved and after a 1-year follow-up, he remained asymptomatic. Conclusion: Even though some authors describe cervical pain associated with this condition, we found very few data regarding plexopathy or other neurological symptoms caused by a cervical transverse apophysomegaly.

Keywords: Cervical, Plexopathy, Spine, Transverse mega-apophysis.

International Journal of Head and Neck Surgery (2021): 10.5005/jp-journals-10001-1418
\end{abstract}

\section{INTRODUCTION}

Cervical transvers mega-apophysis represents a variation of normal skeletal anatomy. This variation had been little studied and its prevalence in the population is unknown because it often exists without symptoms. We present a rare case of a man with a neurogenic thoracic outlet syndrome with left plexopathy caused by a cervical transverse mega-apophysis compression.

\section{Case Description}

A 43-year-old man was referred with limitation in the extension of the first finger of the left hand for 2 years. Afterward, he presented progressive deterioration until developing paresis in flexion of the fingers, in addition to atrophy of the intrinsic musculature of the hand. The patient refers he has paresthesias in the inner part of the arm corresponding to the $\mathrm{C} 8$ and $\mathrm{T} 1$ dermatomes. The patient does not present cervical pain. The pulses in the left arm were normal. There was no clinical evidence of compression of the subclavian

\footnotetext{
${ }^{1-3}$ Department of Neurosurgery, Fundación Jiménez Díaz University Hospital, Madrid, Spain

Corresponding Author: Pierre Ferrer, Department of Neurosurgery, Fundación Jiménez Díaz University Hospital, Madrid, Spain, Phone: +34 9155048 00, e-mail: pierre.ferrer@quironsalud.es

How to cite this article: Ferrer P, Álvarez AS, Penanes JR. Cervical Transverse Mega-apophysis: A Rare Cause of Plexopathy. Int J Head Neck Surg 2021;12(1):40-42.

Source of support: Nil

Conflict of interest: None
}

artery. With Adson's test, the patient refers a little increasement of paresthesias in the inner part of the arm, but the strength of the radial pulse remained similar.

Images show a left C7 transverse mega-apophysis involving the inferior trunk of the brachial plexus at the level of the first costal arch (Figs 1 and 2). There is no existence of a cervical rib.
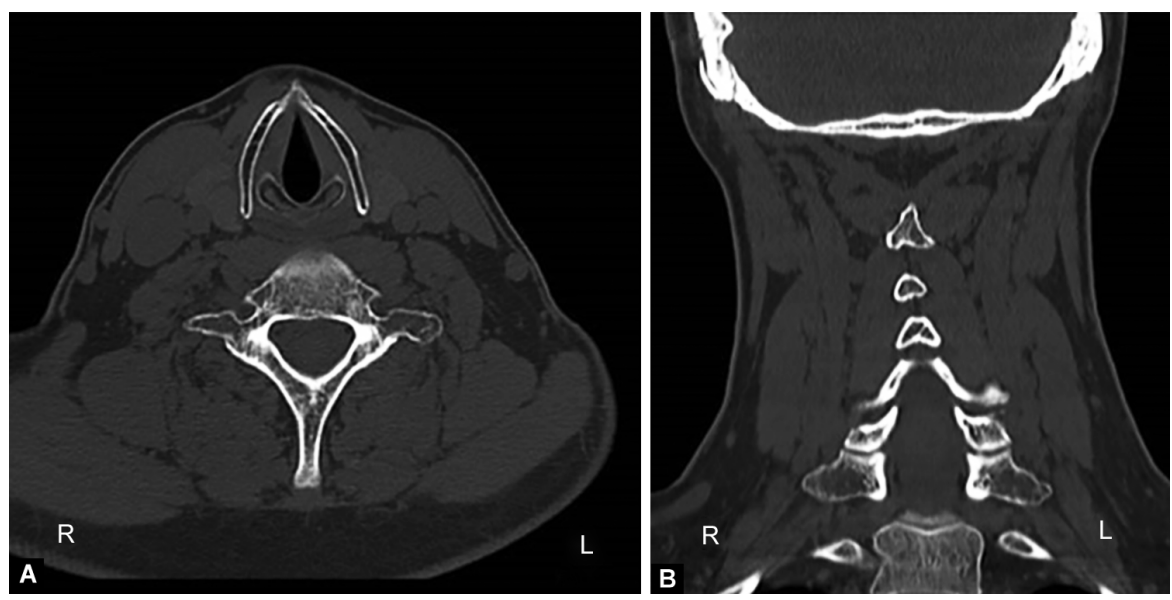

Figs 1 A and B: Preoperative CT scan. Cervical CT axial (A) and coronal (B) view shows left C7 transverse mega-apophysis

\footnotetext{
(c) The Author(s). 2021 Open Access This article is distributed under the terms of the Creative Commons Attribution 4.0 International License (https:// creativecommons.org/licenses/by-nc/4.0/), which permits unrestricted use, distribution, and non-commercial reproduction in any medium, provided you give appropriate credit to the original author(s) and the source, provide a link to the Creative Commons license, and indicate if changes were made. The Creative Commons Public Domain Dedication waiver (http://creativecommons.org/publicdomain/zero/1.0/) applies to the data made available in this article, unless otherwise stated.
} 
The EMG shows a pattern of active denervation in left $\mathrm{C} 8$ and T1 roots.

Our hypothesis was that the inferior trunk of the brachial plexus was compressed between the left C7 transverse megaapophysis and the first left costal arch causing a neurogenic thoracic outlet syndrome. Surgical intervention consisted in a C7 left transversectomy and resection of the first costal arch. After the intervention, the patient improved in left hand paresis and left arm paresthesias (Fig. 3). After 1 year of follow-up, the patient remained asymptomatic.

\section{Discussion}

Congenital cervical skeletal anomalies are not frequent. Transverse mega-apophysis (also named transverse apophysomegaly or elongation of the transverse vertebral process) is defined as an elongation of the transverse process more laterally than that of $\mathrm{T} 1$ when the patient's head is central. It can be unilateral or bilateral and is more frequent at the C7 level. Cervical transverse megaapophysis is identified approximatively in $2 \%$ of the population. However, its prevalence in the population can be greater because it often exists without symptoms and is most commonly an incidental radiographic finding. ${ }^{1-4}$
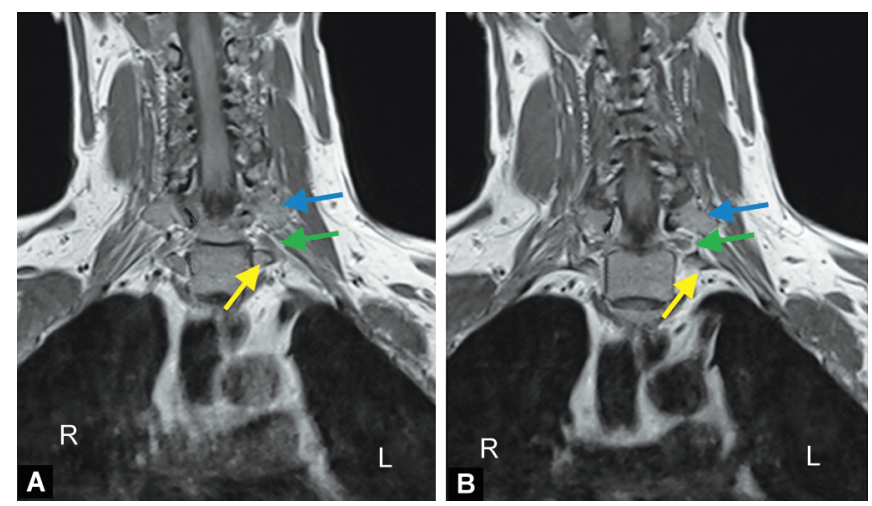

Figs $2 \mathrm{~A}$ and B: Preoperative MRI. Two consecutive sections of a coronal T1-weighted without contrast. MRI shows a left C7 transverse megaapophysis (blue arrow) involving the C8 root (green arrow) at the level of the first left costal arch (yellow arrow)
It is estimated that less than $10 \%$ of cases are symptomatic. Onset of the symptoms is usually in adult life due to the tendency to dropping of the shoulder girdle and the reduction in the elasticity of the tissues. Some maneuvers like Adson maneuver or hyperabduction of the arm may cause the symptoms. Symptoms result from compression of the subclavian artery or the brachial plexus, causing, respectively, arterial thoracic outlet syndrome (ATOS) or neurogenic thoracic outlet syndrome (NTOS), being the C8 and $\mathrm{T} 1$ roots the neural structures most commonly involved causing radiculopathy or plexopathy. There is no correlation between the severity of the clinical impairment and the radiological findings. ${ }^{1,2,5}$

Another congenital cervical skeletal anomaly that is mandatory to differentiate is the cervical rib. This entity varies in comparison to the cervical mega-apophysis because the origin of the compression of the neurovascular structures is between the scalene muscles and the cervical rib rather than being between the first costal arch and the transverse mega-apophysis. ${ }^{6,7}$

Although a conservative treatment should be first in line of therapy, surgical decompression to remove the transverse megaapophysis is recommended when conservative management has failed. ${ }^{7,8}$

\section{Conclusion}

Neurogenic thoracic outlet syndrome is a well-described disorder typically caused by the presence of fibrous and muscular bands, variations in the insertion of the pectoralis minor, the presence of neural structures that follow an atypical course, the existence of a cervical rib, trauma injuries, repetitive arm and shoulder movements and activity, such as from playing certain sports or others causes that can alter the upper thoracic aperture.

However, significantly less literature is found about symptomatic transverse mega-apophysis at a cervical level. Even though some authors describe cervical pain associated with this condition, we found very few data regarding plexopathy caused by cervical transverse mega-apophysis compression in the existing literature.

\section{References}

1. Pepper T, Singh M, Brennan PA. The transverse mega-apophysis - an unusual neck lump. Br J Oral Maxillofac Surg 2010;48(3):201-202. DOI: 10.1016/j.bjoms.2009.08.026.

2. Pionnier R, Depraz A. Congenital rib abnormalities; Statistical study of 10,000 radiographs. Radiol Clin 1956;25:170-186.

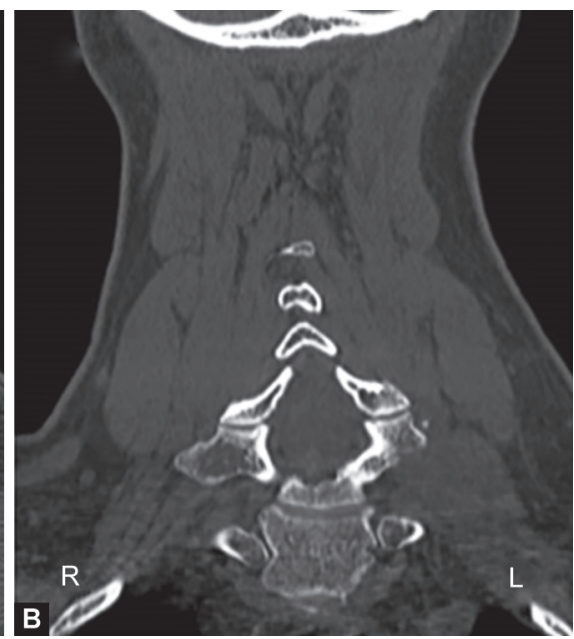

Figs 3A and B: Postoperative CT scan. Cervical CT axial (A) and coronal (B) view shows left C7 transversectomy 
3. Köknel Talu G. Thoracic outlet syndrome. Agri 2005;17(2):5-9.

4. Klaassen Z, Sorenson E, Tubbs RS, et al. Thoracic outlet syndrome: a neurological and vascular disorder. Clin Anat 2014;27(5):724-732. DOI: 10.1002/ca.22271.

5. Boles JM, Missoum A, Mocquard Y, et al. Familial anomaly of the seventh cervical vertebra. Radio-clinic comparison in a fourteenmember family. J de Radiolo 1982;63(4):273-277.

6. Palma A, Carini F. Variation of the transverse apophysis of the 7 th cervical vertebra: anatomo-radiological study of an isolated population. Arch Ital Anat Embriol 1990;95(1): 11-16.

7. Paton GJ, Billings BK. Cervical rib synostosis to the first rib: a rare anatomic variation. World Neurosurg 2020;10(138):187-192. DOI: 10.1016/j.wneu.2020.03.009.

8. Hawks C, Herrera-Nicol S, Pruzansky ME, et al. Minimally invasive resection of symptomatic cervical rib for treatment of thoracic outlet syndrome. World Neurosurg 2020;139:219-222. DOI: 10.1016/ j.wneu.2020.03.049. 